\title{
Interactions of Cationic Lipids with DNA: A Structural Approach
}

\author{
2 Matthias Dittrich, ${ }^{\dagger}$ Chris Brauer, ${ }^{\dagger}$ Sergio S. Funari, ${ }^{\ddagger}$ Bodo Dobner, ${ }^{\S}$ Gerald Brezesinski, ${ }^{*}{ }^{\dagger}$ \\ 3 and Christian Wölk*,§® \\ $4{ }^{\dagger}$ Max Planck Institute of Colloids and Interfaces, Science Park Potsdam-Golm, Am Mühlenberg 1, 14476 Potsdam, Germany \\ 5 "Photon Science - DESY, Notkestrasse 85, 22607 Hamburg, Germany \\ ${ }_{6}{ }^{\S}$ Institute of Pharmacy, Research Group Biochemical Pharmacy, Martin-Luther-University, Wolfgang-Langenbeck-Strasse 4, 06120 \\ 7 Halle (Saale), Germany
}

\begin{abstract}
8 ABSTRACT: Colloidal nucleic acid carrier systems based on cationic lipids are a promising pharmaceutical tool in the implementation of gene therapeutic strategies. This study demonstrates the complex behavior of DNA at the lipidsolvent interface facilitating structural changes of the lyotropic liquid-crystalline phases. For this study, the structural properties of six malonic acid based cationic lipids were determined using small- and wide-angle X-ray scattering (SAXS and WAXS) as well as differential scanning calorimetry (DSC). Selected lipids (lipid 3 and lipid 6) with high nucleic acid transfer activity have been investigated in detail because

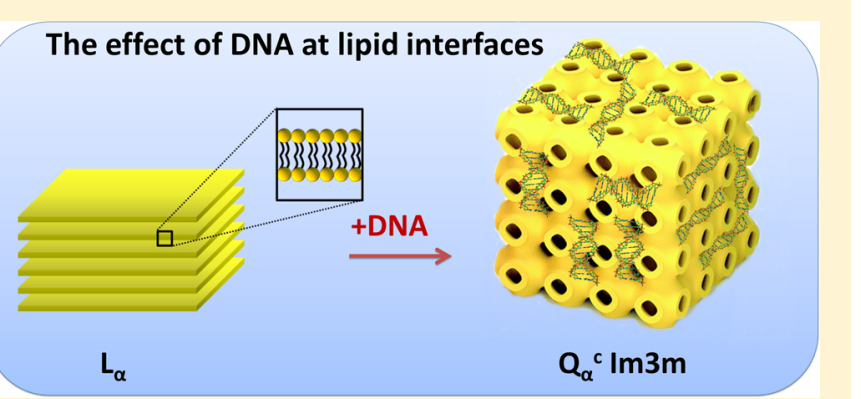
of the strong influence of the zwitterionic helper lipid 1,2-di(9Z-octadecenoyl)-sn-glycero-3-phosphoethanolamine (DOPE) on the structural properties as well as of the complex formation of lipid-DNA complexes (lipoplexes). In the case of lipid 3, DNA stabilizes a metastable cubic mesophase with $\operatorname{Im} 3 m$ symmetry and an $\operatorname{Im} 3 m \mathrm{Q}_{\alpha}{ }^{\mathrm{c}}$ lipoplex is formed, which is rarely described for DNA lipoplexes in literature. In the case of lipid 6, a cubic mesophase with $\operatorname{Im} 3 m$ symmetry turns into a fluid lamellar phase while mixing with DOPE and complexing DNA.
\end{abstract}

\section{INTRODUCTION}

24 Cationic lipid-DNA complexes (lipoplexes) are used as 25 vectors for efficient gene transfer into cells. ${ }^{1-3}$ To obtain 26 outstanding systems, novel lipids are constantly synthesized 27 and new formulations are persistently tested. ${ }^{4-8}$ To increase 28 transfection rates, the cationic lipid structure is varied, the ratio 29 with uncharged helper lipids is changed, different amounts of 30 DNA are added, and lipoplexes are tested in systematical 31 studies (e.g., Heinze et al. ${ }^{9}$ or Wölk et al. ${ }^{10}$ ). ${ }^{11}$ Usually, only a 32 few lipoplexes turn out to be successful gene delivery systems. 33 In this case, it is important to investigate the physical34 chemical properties of the lipids in order to understand the 35 correlations to gene transfer rates. On the basis of complete 36 characterizations of lipids and vectors, successful lipoplexes can 37 be designed more easily in the future.

38 Especially important is the supramolecular assembly of lipids 39 and lipoplexes in bulk. This topic is usually approached by X40 ray scattering experiments. The first pioneering work was done 41 by Rädler et al. with the finding of lamellar lipid-DNA 42 complexes $\left(\mathrm{L}_{\alpha}{ }^{\mathrm{c}}\right)$ as early as 1997. ${ }^{12}$ One year later, the 43 existence of inverted hexagonal structures $\left(\mathrm{H}_{\mathrm{II}}{ }^{\mathrm{c}}\right)$ was 44 reported, ${ }^{13}$ followed by the description of hexagonal structured 45 lipoplexes $\left(\mathrm{H}_{\mathrm{I}}{ }^{\mathrm{c}}\right) \cdot{ }^{14}$ It is believed that by dependence on these 46 structures different transfection rates can be achieved. ${ }^{2}$ Lately, 47 the discussion focuses also on complex structures, e.g., cubic 48 lipid-DNA complexes and mixed phases. ${ }^{15-18}$ Recently, a 49 breakthrough was achieved by Koynova et al. with a review concerning over 30 cationic phospholipids. ${ }^{1}$ The authors were 50 able to show that not only the simple adaption of the $\mathrm{H}_{\mathrm{II}}{ }^{\mathrm{c}} 51$ structure results in an efficient gene transfection ${ }^{13,19}$ but also 52 the pathway into the cell and structural changes on this path 53 seem to be important. For example, lamellar lipoplexes work 54 very well if upon contact with (model) cell membranes a 55 lamellar to nonlamellar phase transition occurs. ${ }^{1}$ In summary, 56 structural properties of lipids and lipoplexes cannot be related 57 to transfection rates in a straightforward manner for all cases. 58 Moreover, it is important to understand and explain each single 59 case in order to identify different promising structures and 60 mechanisms.

This work focuses on the physical-chemical characterization 62 of six lipids designed for gene transfection (Figure 1). $63 \mathrm{f} 1$ Synthesis and transfection results are already described. ${ }^{10} 64$ Lipids 1-3 and 4-6 possess different headgroups. The lipid 65 chains are either saturated or unsaturated. Lipids $\mathbf{1}$ and $\mathbf{2}$ are 66 not able to incorporate colipids and show no transfection 67 activity. ${ }^{10}$ Lipids 3 and $\mathbf{6}$ are the most promising gene transfer 68 vehicles in complexes with noncharged colipids and DNA. The 69 mixtures of lipid 6 with DOPE 2:1 (n/n) and lipid 3 with 70 DOPE 1:2 (n/n) exhibit transfection efficiencies higher than 71

Special Issue: Nucleic Acids Nanoscience at Interfaces

Received: May 16, 2018

Revised: July 21, 2018

Published: August 30, 2018 
<smiles>[R7]NC(=O)C([R2])C(=O)NCCN(CCN)CCNC(=O)C(N)CCCCN</smiles>

Figure 1. Chemical structures of lipids 1-6.

72 those of commercially available transfection reagents in cell 73 culture models even in the presence of serum. ${ }^{10}$ The lipids 4 74 and $\mathbf{5}$ showed moderate transfection efficiency. ${ }^{10}$

75 The article by Wölk et al. also presents some preliminary 76 data about the phase behavior of these lipids. ${ }^{10}$ However, the 77 data required more detailed investigations to reveal structural 78 characteristics which determine the degree of transfection 79 efficiency, and this is the topic of the present work. In this 80 work, supramolecular structures and thermodynamic proper81 ties of lipids $\mathbf{1 - 6}$ in bulk are investigated by X-ray scattering 82 and DSC. The phase structures of the pure lipids as well as of 83 the selected lipid mixtures, which are especially promising gene 84 transfer systems, have been determined. The physical85 chemical properties have been connected with the transfection 86 results described earlier. ${ }^{10}$ The lipoplex formation between the 87 lipid mixtures and DNA changes the supramolecular structures, 88 demonstrating the complex interaction behavior between DNA 89 and cationic lipids at the interface.

\section{EXPERIMENTAL SECTION}

90 2.1. Materials. For all measurements and sample preparations, 91 Milli-Q Millipore water with a specific resistance of $18.2 \mathrm{M} \Omega \cdot \mathrm{cm}$ was 92 used. DNA from calf thymus (product number D1501) was 93 purchased from Sigma-Aldrich. The helper lipid 1,2-dioleoyl-sn94 glycero-3-phosphoethanolamine (DOPE) was purchased from Avanti 95 Polar Lipids. The synthesis and purification of the cationic lipids were 96 described earlier. ${ }^{10}$ All other chemicals were of analytical grade and 97 used without further purification.

98 2.2. Differential Scanning Calorimetry (DSC). Lipids were 99 dispersed in Milli-Q water to a concentration of $1 \mathrm{mg} \cdot \mathrm{mL}^{-1}$. After 100 hydration, the aqueous dispersions were vortexed, sonicated, and 101 heated (usually above the phase transition temperature of the 102 corresponding lipid) several times. DSC measurements were 103 performed on a microcalorimetry system (MCS DSC, MicroCal 104 Inc., Northampton, MA). The sample and the reference were 105 degassed for at least $20 \mathrm{~min}$ by stirring under a vacuum. After filling, 106 the DSC cells were kept under 2 bar of nitrogen pressure to prevent 107 evaporation at higher temperatures. The heating rate was $60 \mathrm{~K} \cdot \mathrm{h}^{-1}$. 108 Samples were scanned at least $4 \times$ up to $\approx 85{ }^{\circ} \mathrm{C}$ after being cooled to $10910{ }^{\circ} \mathrm{C}$ to prove reproducibility.

110 For data treatment, the first heating scan was neglected. The main 111 transition temperature from gel to liquid-crystalline state $T_{\mathrm{m}}$ was 112 determined as the maximum of the corresponding heat flow curves. 113 Transition enthalpies $\Delta H$ (main phase transition enthalpy $\Delta H_{\mathrm{m}}$ and 114 pretransition enthalpy $\left.\Delta H_{\mathrm{p}}\right)$ were determined by numerical integration after baseline correction. An estimated error of about 115 $10 \%$ has to be taken into account for transition enthalpies. 116

2.3. X-ray Scattering. Lipids and lipid mixtures were prepared as 117 described in section 2.2. Lipid concentrations of $200 \mathrm{mg} \cdot \mathrm{mL}^{-1}$ in 118 Milli-Q water were used. Lipid-DNA complexes were prepared by 119 adding a $2 \mathrm{mg} \cdot \mathrm{mL}^{-1}$ DNA solution to the solid lipid mixture in the 120 corresponding N/P ratio (ratio of moles of primary amine groups of 121 cationic lipid to phosphate groups of DNA). The immediately formed 122 complex was vortexed and sonicated for at least $15 \mathrm{~min}$. If necessary, 123 samples were centrifuged. Finally, the supernatant was separated from 124 the lipoplex with a micropipette. Afterward, samples containing DNA 125 were heated $\left(2 \mathrm{~K} \cdot \mathrm{min}^{-1}\right)$ once up to $60{ }^{\circ} \mathrm{C}$.

All samples were transferred into open glass capillaries (inner 127 diameter $1.5 \mathrm{~mm}$, GLAS). A flexible tube was used to connect a 128 syringe to one open end of the capillary. The dispersions were sucked 129 in, and the capillary was sealed on both sides with a Microtorch 130 device. Samples were stored for 7-10 days at $4{ }^{\circ} \mathrm{C}$ before 131 measurements.

Synchrotron small-angle (SAXS) and wide-angle X-ray scattering 133 (WAXS) experiments were carried out at the Soft Condensed Matter 134 beamline A2, HASYLAB, DESY (Hamburg, Germany). The standard 135 procedures that were used have been described elsewhere. ${ }^{20-23}$ SAXS 136 and WAXS data were taken simultaneously by a MAR CCD detector 137 (Evanston, Illinois, USA) and a linear detector with delay line 138 readout, respectively. The incoming beam had a wavelength of 0.15139 $\mathrm{nm}$, and the exposure time was between 20 and $300 \mathrm{~s}$. The 140 temperature was fixed during the measurements. After each heating 141 step $\left(2 \mathrm{~K} \cdot \mathrm{min}^{-1}\right)$, a waiting time of $5 \mathrm{~min}$ was used to ensure that the 142 sample was in thermal equilibrium. Dry rat tail collagen (SAXS) and 143 polyethylene terephthalate (WAXS) were used for calibration. 144 Positions of the peaks were converted into reciprocal spacings $s=145$ $1 / d$, with $d$ being the real space repeat distance of the lattice planes. In 146 order to determine peak maxima and the full-width at half-maximum 147 (fwhm) $\Delta s$, the experimental points were fitted with a Lorentzian 148 function. Overlaying diffraction peaks were fitted by a superposition 149 of Lorentzians (including diffuse scattering). Correlation lengths $\xi 150$ were calculated using $\xi=1 / \Delta s$ of the first-order diffraction peak. 151

Lamellar phases can be identified by equidistant peaks in the SAXS 152 patterns. In this case, the lamellar repeat distance $d$ (lipid bilayer +153 one adjacent layer of water) can be calculated using $d=1 / s$. The 154 relative peak positions of hexagonal and inverted hexagonal phases are 155 given by $\sqrt{h^{2}+k^{2}-h k}(1, \sqrt{3}, \sqrt{ } 4, \sqrt{ } 7, \sqrt{9}, \ldots)$, with $h$ and $k 156$ being the Miller indices that characterize a set of lattice planes. The 157 lattice parameter $a$ for the hexagonal unit cell can be calculated using 158 $a=\frac{2 \sqrt{h^{2}+k^{2}-h k}}{\sqrt{3} s_{h k}}$. Cubic liquid-crystalline lattices show spacings in the ratio of $\sqrt{h^{2}+k^{2}+l^{2}}(1, \sqrt{ } 2, \sqrt{ } 3, \sqrt{ } 4, \sqrt{ } 5, \sqrt{ } 6, \sqrt{ } 9, \ldots)$, with $h, k, 159$ and $l$ being the Miller indices that characterize a set of lattice planes. 160 The absence of peaks determines the lattice symmetry. Typical phases 161 are $I a 3 d, P n 3 m, I m 3 m$, and $P m 3 n$ (see Lindblom et al. ${ }^{2}$ for details). 162 To identify cubic phases, the experimentally obtained peak positions $s \quad 163$ are plotted as a function of the sum of the Miller indices to which the 164 peaks are indexed. A linear function with an $(0,0)$ intercept is 165 generated. The average lattice parameter $a$ equals the inverse slope of 166 this function.

167

\section{RESULTS AND DISCUSSION}

3.1. DSC with Pure Lipids. Figure 2 shows the $168 \mathrm{f} 2$ thermograms of lipids 1-6. The transition temperatures and 169 enthalpies are given in Table 1 . The DCS curves of lipids 1 and 170 t1 6 have already been presented in previous work ${ }^{10}$ but are 171 shown here again for comparison.

Lipids 3, 4, and 6 show no phase transitions in the accessible 173 temperature range. Lipid $\mathbf{5}$ shows a transition with a small 174 $\Delta H_{\mathrm{m}}$ at low temperatures. Lipid 2 reveals a slightly higher $T_{\mathrm{m}} 175$ with a similar value for $\Delta H_{\mathrm{m}}$. In contrast, the thermogram of 176 lipid 1 shows a pretransition and a main phase transition at 177 


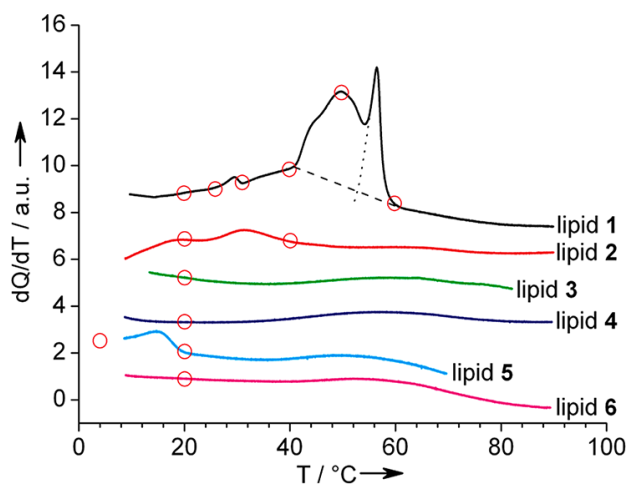

Figure 2. DSC thermograms (heat flow $d Q / d T$ as a function of $T$ ) of lipids 1-6. The red circles indicate the temperatures where X-ray data were taken. The dotted line represents an approximated separation of the two phase transitions of lipid $\mathbf{1}$ and is only to guide the eye. The dashed line defines the area that was integrated to obtain the total $\Delta H_{\mathrm{m}}$ of lipid 1. Curves are shifted vertically for clarity. The DSC curves of lipid $\mathbf{1}$ and lipid $\mathbf{6}$ are taken from the literature. ${ }^{10}$

Table 1. Main Transition Temperatures $T_{\mathrm{m}}$, Main Transition Enthalpies $\Delta H_{\mathrm{m}}$, Pretransition Temperatures $T_{\mathrm{m}}$, and Pretransition Enthalpy $\Delta H_{\mathrm{p}}$ of Lipids 1,2 , and $5^{a}$

\begin{tabular}{cll} 
lipid & $T_{\mathrm{m}}\left[{ }^{\circ} \mathrm{C}\right]$ & $\Delta H_{\mathrm{m}}\left[\mathrm{kJ} \cdot \mathrm{mol}^{-1}\right]$ \\
\hline lipid 1 & $T_{\mathrm{p}}=29.4$ & $\Delta H_{\mathrm{p}}=0.9$ \\
& $49.7,56.5$ & 50.6 \\
lipid 2 & 31.4 & 5.1 \\
lipid 5 & 14.9 & $\sim 4.4$
\end{tabular}

${ }^{a}$ The values are determined from the DSC curves presented in Figure 2. Transitions outside the investigated temperature window might be possible but cannot be determined from the presented experiments.

178 high temperatures. The latter can be divided into a broad peak 179 and a sharp transition peak. $\Delta H_{\mathrm{m}}$ of the complete transition is $18050.6 \mathrm{~kJ} \cdot \mathrm{mol}^{-1}$ which is high compared to those of well-known 181 lipids with similar chain lengths as dipalmitoylphosphatidyl182 choline (DPPC) and dipalmitoylphosphatidylethanolamine 183 (DPPE) that exhibit main phase transition enthalpies between 18435 and $40 \mathrm{~kJ} \cdot \mathrm{mol}^{-1} \cdot{ }^{25,26}$ It is reasonable to assume that the 185 high $\Delta H_{\mathrm{m}}$ is not only a result of the chain melting in that 186 region. Lipids with similar structures exhibit similar high 187 transition enthalpies. ${ }^{27}$ Contributions made by the breaking of 188 hydrogen bonds between headgroups are discussed. Hydration 189 of lipid heads might need additional energy as well and is 190 discussed in sections 3.2 and 3.3.

191 Lipids 1-3 have the same headgroup but different chain 192 patterns. The decrease of $T_{\mathrm{m}}$ and $\Delta H_{\mathrm{m}}$ is clearly connected 193 with the insertion of double bonds into the lipid chains (lipid 194 1, $\mathrm{C}_{16}$ and $\mathrm{C}_{16}$; lipid 2, $\mathrm{C}_{18: 1}$ and $\mathrm{C}_{16}$; lipid 3, $\mathrm{C}_{18: 1}$ and $\mathrm{C}_{18: 1}$ ). 195 The extension of the saturated chains would lead to an increase 196 of the gel to liquid-crystalline transition temperature and 197 enthalpy as observed, e.g., for phosphatidylcholines. ${ }^{28}$ In the 198 present case, the chain length increase is overcompensated by 199 the introduction of the double bond which needs more space 200 due to steric hindrance. Therefore, van der Waals interactions 201 are reduced and lipids with more double bonds are found to 202 have much lower phase transition temperatures, allowing lipid 2033 to be in the liquid-crystalline state already at room 204 temperature.

205 In the case of lipids 4-6 (the headgroup is more bulky than 206 in the case of lipids $\mathbf{1 - 3}$ ), the tendency is not so 207 straightforward. The insertion of one double bond leads to an apparent phase transition in the case of lipid $\mathbf{5}\left(\mathrm{C}_{18: 1}, \mathrm{C}_{16}\right) .208$ In contrast, lipid $4\left(\mathrm{C}_{16}, \mathrm{C}_{16}\right)$ and lipid $\mathbf{6}\left(\mathrm{C}_{18: 1}, \mathrm{C}_{18: 1}\right)$ show no 209 phase transition in the accessible temperature range. The large 210 headgroup of this series seems to be the key factor governing 211 the phase state. The mismatch in the area requirement of the 212 chains and of the headgroup of lipid 4 disables favorable van 213 der Waals interactions between the neighboring chains. Maybe 214 the combination of one oleyl and one hexadecyl chain in 215 mixed-chain lipids with large headgroups leads to increased 216 chain-chain interactions due to more efficient packing with 217 fewer defects, thereby enhancing the van der Waals contacts as 218 compared to those of the analogues with two hexadecyl or two 219 oleyl chains, and the phase transition of lipid $\mathbf{5}$ is in the 220 accessible temperature range. Finally, the strong disorder 221 induced by two double bonds leads again to the observed 222 decrease of the transition temperature.

223

The correlation of DSC experiments with transfection 224 results suggests that especially membrane fluidity plays an 225 important role. While lipids $\mathbf{1}$ and $\mathbf{2}$ are still in the gel phase at 226 room temperature, the other four lipids are in the liquid- 227 crystalline state. This statement was mentioned in previous 228 work ${ }^{10}$ but is proved in the present article by DSC and WAXS 229 experiments (this section and section 3.2). The inability of 230 lipids 1 and 2 to mix with noncharged helper lipids which are 231 in the liquid-crystalline state seems to be connected with the 232 immobility of the lipid chains in the gel state. ${ }^{52,53}$ According to 233 Sackmann and Demus, two compounds never mix completely 234 if they form different phase structures. ${ }^{54}$ An important role can 235 be, therefore, ascribed to the degree of saturation which is 236 closely related to the phase state and seems to be connected 237 with the efficiency data published earlier. ${ }^{10}$ This work confirms 238 former studies that were also able to show that especially $\mathrm{C}_{18: 1} 239$ chains are more suitable for gene transfection. ${ }^{4,7,27,29}$ Also the 240 headgroup affects the efficiency. A certain increase in gene 241 transfer activity has even been observed for lipids with two 242 saturated chains by increasing the size of the headgroup (lipid 243 $4 \rightarrow$ weak transfection compared to lipid $\mathbf{1} \rightarrow$ no 244 transfection). ${ }^{10}$ Obviously the increase in the space require- 245 ment of the headgroup reduces the chain-chain interactions. 246 Nevertheless, the introduction of oleyl chains has more 247 pronounced positive effects on the transfection efficiency. 248 The delicate interplay between hydrophobic and hydrophilic 249 parts is obviously very important for successful transfection. In 250 any case, the most promising gene transfer results were 251 obtained with lipids 3 and $\mathbf{6}$ with high degrees of unsaturation 252 and, therefore, high fluidities.

253

3.2. WAXS Patterns of Pure Lipids. Figure 3 shows $254 \mathrm{f3}$ WAXS patterns of all lipids at selected temperatures according 255 to the DSC thermograms. If no phase transition was observed, 256 only one experiment was performed at $20{ }^{\circ} \mathrm{C}$ to identify the 257 phase state. The scattering curves of lipid 1 at 20 and $60^{\circ} \mathrm{C}$ as 258 well as the curve of lipid 6 have been already published ${ }^{10}$ but 259 shown here again for comparison. The chain lattice parameters 260 are given in Table 2 .

$261 \mathrm{t} 2$

A broad halo in the WAXS region at $20^{\circ} \mathrm{C}$ indicates that the 262 lipids 3, 4, 5, and 6 are in the liquid-crystalline state $\left(\mathrm{L}_{\alpha}\right)$ (see 263 section 3.1) and proves the assumptions that were made 264 earlier. ${ }^{10}$ The small remaining peak of lipid $\mathbf{4}$ is discussed in 265 section 3.3. For lipid 5, the gel state can be reached at 266 temperatures $\leq 10{ }^{\circ} \mathrm{C}$. At $20^{\circ} \mathrm{C}$, the corresponding gel peak 267 with weak intensity is still present, showing that the phase 268 transition is not yet completed. An orthorhombic lattice with 269 

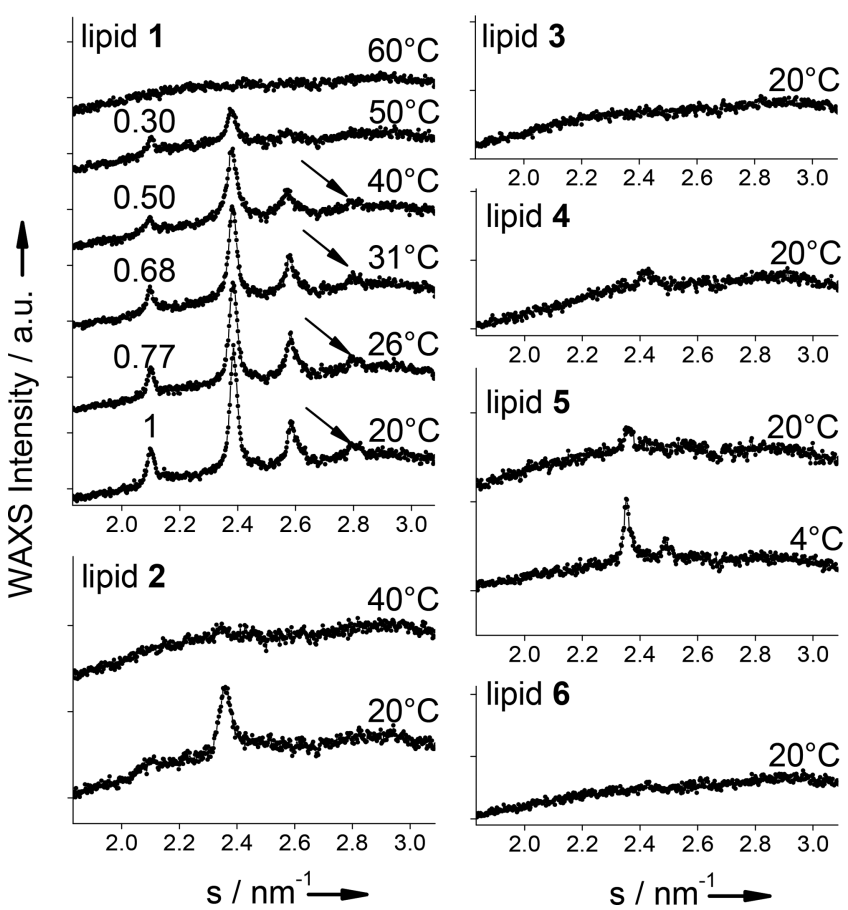

lipid 4
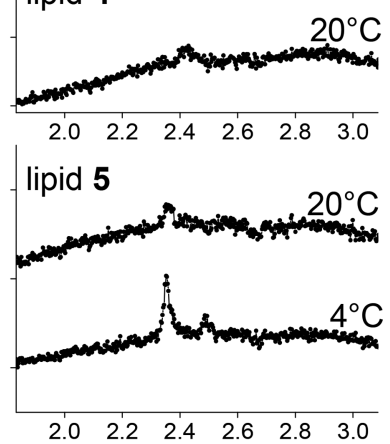

lipid 6

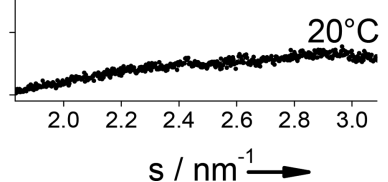

Figure 3. WAXS patterns of lipids 1-6 at selected temperatures. The arrows indicate a Bragg peak that is not arising from the chain lattice of lipid 1. Numbers indicate the relative intensities (normalized after integration to the intensity observed at $20{ }^{\circ} \mathrm{C}$ ) of the hydrogen bond peak of lipid 1 after baseline correction. Curves are shifted vertically for clarity. The scattering curves of lipid 1 at 20 and $60{ }^{\circ} \mathrm{C}$ as well as of lipid $\mathbf{6}$ are taken from literature. ${ }^{10}$

Table 2. Peak Positions $s$ and Cross-Sectional Areas $A$ of Lipids $1-6$ at Different Temperatures $T$ Derived from the WAXS Data

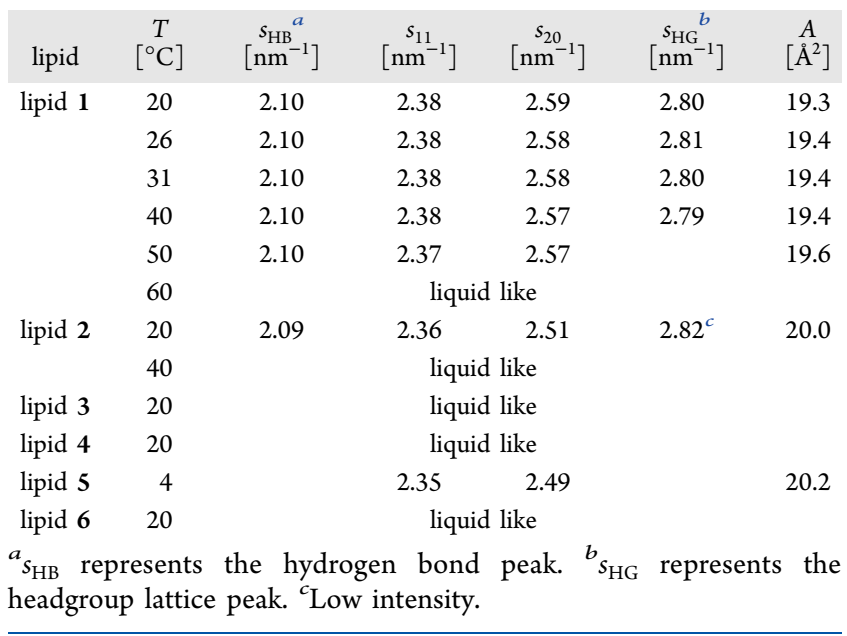

270 tilted chains $\left(\mathrm{L}_{\mathrm{B}^{\prime}}\right)$ and a cross-sectional area typical of gel 271 phases can be found at $4{ }^{\circ} \mathrm{C}$.

272 The WAXS patterns of lipids $\mathbf{1}$ and $\mathbf{2}$ show more details than 273 those of the other lipids. At $50{ }^{\circ} \mathrm{C}$ (below the sharp phase 274 transition), a Bragg peak at $s_{\mathrm{HB}} \sim 2.10 \mathrm{~nm}^{-1}(d=0.476 \mathrm{~nm})$ is 275 an indication of hydrogen bonds between the lipid headgroups. 276 This peak was first assigned to hydrogen bonds between N$277 \mathrm{H} \cdots \mathrm{O}=\mathrm{C}$ groups of $B$-sheets of proteins. ${ }^{30-32}$ Lately, this has 278 been discussed with regard to headgroup interactions of lipids 279 with similar structures as well. ${ }^{27,33}$ The lattice structure of lipid 2802 below $T_{\mathrm{m}}$ is gel-like $\left(\mathrm{L}_{B^{\prime}}\right)$ whereas the packing of the chains of lipid $\mathbf{1}$ is very tight and suggests a subgel phase $\left(\mathrm{L}_{\mathrm{c}}\right)$. A unit 281 cell of $5.0 \times 7.7 \AA^{2}$ at $20{ }^{\circ} \mathrm{C}$ can be compared to the well- 282 known herringbone arrangement of hydrocarbon chains in 283 organic crystals $\left(5.0 \times 7.5 \AA^{2}\right) .{ }^{34}$ Another peak is found at 284 higher $s$ values (arrows in Figure 3), which cannot be assigned 285 to the chain lattice. Its origin is based on an independently 286 formed headgroup lattice ( $s_{\mathrm{HG}}$ peak). Unfortunately, this peak 287 was the only one available in the range of the detector. A 288 headgroup lattice structure can, therefore, not be derived. It 289 should be mentioned that a similar structure is obtained by 290 grazing incidence X-ray diffraction experiments at the air- 291 water interface. $^{33}$ The accessible $s$-range is much larger, 292 allowing the observation of a number of scattering peaks 293 which allowed identification of the lattice structure.

294

The lattice structure of lipid $\mathbf{1}$ does not change upon 295 increasing the temperature to $40{ }^{\circ} \mathrm{C}$. Thus, the pretransition 296 found in the DSC thermogram cannot be assigned to the 297 rearrangement of the chain lattice. At $50{ }^{\circ} \mathrm{C}, s_{\mathrm{HG}}$ and the other 298 peaks decrease strongly in their intensities. The chains start to 299 melt at temperatures above $40{ }^{\circ} \mathrm{C}$ and not only during the 300 second transition (sharp DSC peak at $56.5^{\circ} \mathrm{C}$ ). Because of the 301 tight chain packing of lipid $\mathbf{1}$ and the additional headgroup 302 interactions, water molecules cannot interact easily with the 303 lipid heads at low temperatures. With increasing temperature, 304 the $s_{\mathrm{HB}}$ peak decreases continuously in intensity (see relative 305 intensities in Figure 3), showing increasing disorder in the 306 headgroup region. The loosening of the hydrogen bonds in the 307 headgroup region favors the penetration of water molecules. 308 Therefore, a certain hydration heat is constantly produced, 309 helping to break the hydrogen bonds over a large temperature 310 range. The chains of already fully hydrated lipids (region of the 311 broad DSC transition) start to melt first; the less hydrated 312 lipids follow at higher temperatures. At $60{ }^{\circ} \mathrm{C}$, the chain lattice 313 of lipid 1 disappears completely. The transition from the $\mathrm{L}_{c} 314$ state to a liquid-crystalline state seems to occur without any 315 intermediate phase state. The chains of lipid $\mathbf{2}$ have already 316 melted at $40{ }^{\circ} \mathrm{C}$. It is important to note that the oleylamine 317 from Sigma-Aldrich used for the synthesis of the lipids 318 (oleylamine is bound via an amide bond as $\mathrm{R}_{1}$; see Figure 1) 319 contains only $70 \%$ of the $\mathrm{C}_{18: 1}$ chain and different saturated 320 chains but is a primary amine to $98 \%$. The synthesis and 321 purification lead to a slightly better purity $\left(\mathrm{C}_{18: 1} 81 \mathrm{~mol} \%, 322\right.$ $\mathrm{C}_{18: 0} 6 \mathrm{~mol} \%, \mathrm{C}_{16: 0} 10 \mathrm{~mol} \%$, and $\left.\mathrm{C}_{14: 0} 3 \mathrm{~mol} \%\right) .{ }^{33}$ This 323 oleylamine is used because of the idea of a cheap large scale 324 production of such lipids which are successfully used in 325 transfection experiments. Knowing that, one would expect 326 broader transition ranges for the lipids containing the 327 unsaturated chains.

The WAXS data gives an explanation for the preparation of 329 lipid dispersions (in general mixtures with uncharged helper 330 lipids) used in transfection experiments. Lipid $\mathbf{1}$ shows strong 331 self-association on the basis of interactions between chains and 332 headgroups. The tight packing and the $\mathrm{L}_{\mathrm{c}}$ state are disadvanta- 333 geous for the formation of lipid mixtures which have a charge 334 density suitable for transfection. The helper lipids cannot easily 335 be incorporated into the bilayers of this lipid, and the 336 complexes are, therefore, unstable. Lipid $\mathbf{2}$ is found to be in the 337 gel state but with fewer headgroup interactions due to the 338 larger area required by the chains. However, the inflexibility of 339 the lipid chains seems to be enough to prevent effective 340 incorporation of helper lipids. In contrast, lipids 3-6 which are 341 in the liquid-crystalline state at room temperature exhibit a 342 
Table 3. Structure, Peak Positions $s$, and Calculated Interlamellar Repeat Distance $d$ of Lipids 1-6 at Different Temperatures $T$ Obtained from the X-ray Data

\begin{tabular}{|c|c|c|c|c|c|c|c|}
\hline lipid & $\mathrm{T}\left[{ }^{\circ} \mathrm{C}\right]$ & structure & $s_{001}\left[\mathrm{~nm}^{-1}\right]$ & $s_{002}\left[\mathrm{~nm}^{-1}\right]$ & $s_{003}\left[\mathrm{~nm}^{-1}\right]$ & $s_{004}\left[\mathrm{~nm}^{-1}\right]$ & $d[\mathrm{~nm}]$ \\
\hline \multirow[t]{6}{*}{ lipid 1} & 20 & lamellar, correlated + uncorrelated (brick structure, vesicles) & & 0.108 & 0.158 & 0.210 & 18.8 \\
\hline & 26 & & & 0.109 & 0.158 & 0.211 & 18.7 \\
\hline & 31 & & & 0.110 & 0.162 & 0.211 & 18.5 \\
\hline & 40 & & & 0.116 & 0.170 & 0.222 & 17.6 \\
\hline & 50 & & & 0.119 & 0.176 & 0.232 & 17.0 \\
\hline & 60 & & $0.062^{a}$ & 0.127 & 0.192 & & 15.7 \\
\hline \multirow[t]{4}{*}{ lipid 2} & 10 & lamellar, correlated + uncorrelated (brick structure, vesicles) & & 0.112 & 0.164 & 0.210 & 18.3 \\
\hline & 20 & & & 0.114 & 0.168 & 0.217 & 17.9 \\
\hline & 30 & & & 0.115 & 0.172 & 0.224 & 17.6 \\
\hline & 40 & & & 0.107 & 0.161 & 0.215 & 18.6 \\
\hline lipid 3 & 20 & lamellar & 0.154 & 0.307 & & & 6.50 \\
\hline lipid 4 & 20 & uncorrelated (vesicles) + lamellar & 0.099 & 0.198 & 0.299 & & 10.08 \\
\hline \multirow[t]{2}{*}{ lipid 5} & 4 & lamellar correlated + uncorrelated & 0.183 & 0.366 & & & 5.46 \\
\hline & 20 & & & & & & \\
\hline lipid 6 & 20 & cubic & $=13.42 \mathrm{~nm}$ & & & & \\
\hline
\end{tabular}

343 better ability to incorporate helper lipids and to build mixtures 344 with a charge density suitable for effective transfection. ${ }^{3}$

$345 \quad$ 3.3. SAXS Patterns of Pure Lipids. SAXS patterns reveal 347 structural parameters derived from the X-ray data are 348 summarized. The X-ray patterns for lipids $\mathbf{1}$ and $\mathbf{2}$ are shown 349 in Figure 4.

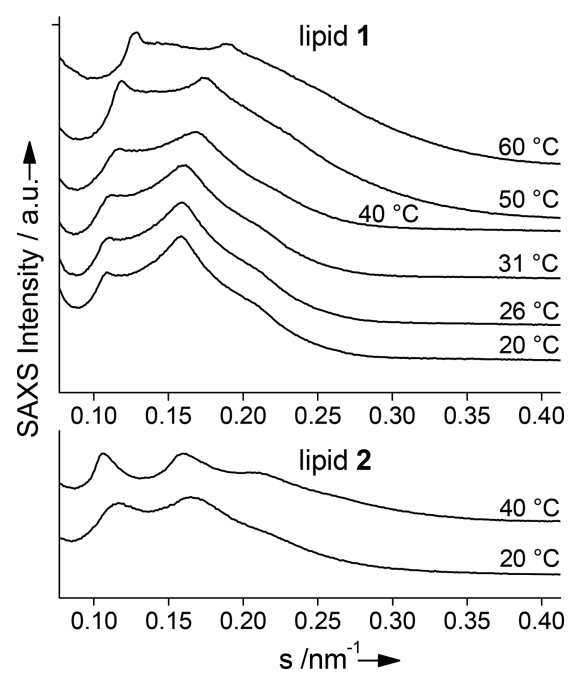

Figure 4. SAXS patterns of lipids 1 and 2 at different temperatures. Curves are vertically shifted for clarity.

350 The profiles show distinct Bragg reflexes which indicate a 351 liquid-crystalline phase in the presence of uncorrelated 352 bilayers, ${ }^{35}$ which result in diffuse scattering (broad back353 ground). The reciprocal spacings of the quasi Bragg peaks are 354 found to be roughly in a ratio of $1: 1.5: 2$ for all diffraction 355 patterns (see Table 3). An equidistant spacing (1:2:3:...) 356 characteristic for lamellar phases can only be achieved if it is 357 assumed that the first-order scattering peak is located at very 358 low $s$ values which are out of the detector range. This is, in fact, 359 the case and can be supported by a small shoulder observed at $360 s=0.062 \mathrm{~nm}^{-1}$ for lipid 1 at $60{ }^{\circ} \mathrm{C}$. This shoulder appears in 361 the intensity profile of the direct beam (not shown).

From the small $s$ values, unusually large $d$ values are 362 calculated (between $\approx 16$ and $\approx 19 \mathrm{~nm}$, see Table 3 ). Because 363 of tight packing in the case of lipid 1 (see section 3.2), it can be 364 expected that chain and headgroups are almost completely 365 stretched. Therefore, the membrane thickness is estimated to 366 be between 7 and $8 \mathrm{~nm}$. The interlamellar repeat distances, $d, 367$ calculated for both lipids are much larger than estimated. The 368 cause can be either that there is a unit cell that does not 369 contain one lipid bilayer but a double bilayer or, what is more 370 plausible, that the large interspace is filled with water and 371 results from strong repulsive forces between the positively 372 charged lipid bilayers. The hypothesis of a unit cell with two 373 lipid bilayers is described in the literature for different systems 374 of mixed lipids. ${ }^{36,37}$ However, one condition is that both 375 bilayers showed different properties: e.g., each one consisted of 376 a different amphiphile. In the present case, structural 377 differences in single component systems are not reasonable 378 whereas the hypothesis of an extended water layer between the 379 lipid bilayers is more favored. Therefore, we consider that the 380 bilayers of both lipids are separated by $\sim 10 \mathrm{~nm}$ of water. $\quad 381$

In order to obtain the electron density profiles (Figure 5), $382 \mathrm{f5}$ the X-ray patterns of lipids $\mathbf{1}$ and $\mathbf{2}$ at $20{ }^{\circ} \mathrm{C}$ were fitted 383 exemplarily according to Pabst. ${ }^{35,38,39}$ The software that was 384 used (Global Analysis Program v. 1.3) allowed the fitting of 385 data that consisted of (quasi) Bragg peaks and diffuse 386 scattering. The fits are in good agreement with the 387 experimental data points and prove that the data can originate 388 from one bilayer with a huge interlayer spacing filled only with 389 water. The membrane thickness (taken at the fwhm of the 390 headgroup Gaussians) is $71 \AA$ for lipid 1 and $64 \AA$ for lipid 2. 391 This difference is in good agreement with the WAXS data. Due 392 to the tight packing of lipid $\mathbf{1}$ molecules, the headgroups are 393 more stretched than the ones of lipid 2 (reduced packing 394 density of the chains). Additionally, the chains of lipid $\mathbf{2}$ have 395 the possibility to interdigitate partially due to the asymmetric 396 chain pattern.

The water-containing interlayer amounts to 122 A for lipid 1398 and $114 \AA$ for lipid 2. We asked why a correlation over such 399 large distances exists so that clear Bragg reflexes appear 400 (without such a correlation only a very broad intensity 401 distribution would be expected) and which driving force is 402 behind it. The fitting parameters suggest that the average 403 


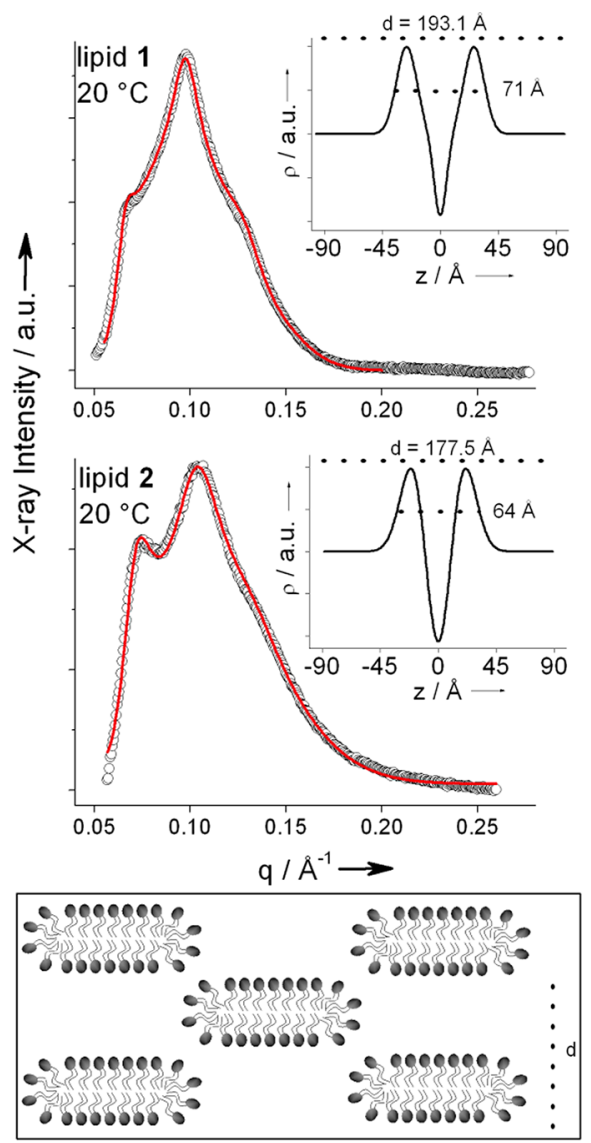

Figure 5. X-ray diffraction patterns $(\mathrm{O})$ and corresponding electron density profiles of lipids $\mathbf{1}$ and 2 at $20{ }^{\circ} \mathrm{C}$. The solid red lines represent the fits. A model that is able to explain the large $d$ values is shown on the bottom.

404 number of bilayers per scattering domain is $\sim 2.5$ for both 405 lipids and the percentage of diffuse scattering is high. However, 406 there are still some bilayers that are correlated over this 407 distance. One explanation might be a brick-like structure of the 408 membranes. Due to strong interactions of head and tail groups, 409 the membranes are very rigid and pack, possibly, as illustrated 410 in Figure 5 without breaking apart. The "bricks" are built due 411 to the large area requirement of the headgroups, unlike the 412 chains, which prohibits an extended lamellar arrangement and 413 leads to the shown structures (no continuous bilayers). Such a 414 model also means that the lipid forms into multilamellar stacks 415 rather than vesicles. Lyotropic phases with large interlamellar 416 water layers have been described ("iridescent phases"). ${ }^{55,56} \mathrm{It}$ 417 is believed that electrostatic forces between charged surfactants 418 are necessary. However, they are too small to explain the 419 stability of the systems. Therefore, it is assumed that the 420 bilayers are strongly fluctuating and are stabilized by steric 421 repulsion.

422 This assumption is supported by the supramolecular 423 structure of lipid 3 at $20{ }^{\circ} \mathrm{C}$. Due to the introduction of a 424 double bond in each chain, a "normal" lamellar phase forms at $42520{ }^{\circ} \mathrm{C}$ (Figure 6). Chains and headgroup now need similar 426 amounts of space. The sharp Bragg peaks with an equidistant 427 spacing of $s_{001}=0.154$ and $s_{002}=0.307 \mathrm{~nm}^{-1}$ indicate a 428 continuous bilayer system with a correlation length that is $\sim 10$ 429 times higher $(\xi=147 \mathrm{~nm})$ than those of lipids 1 and 2 . The 430 lamellar repeat distance is $6.5 \mathrm{~nm}$ and is reasonable for a lipid 431 of that size with chains in $\mathrm{L}_{\alpha}$-state.

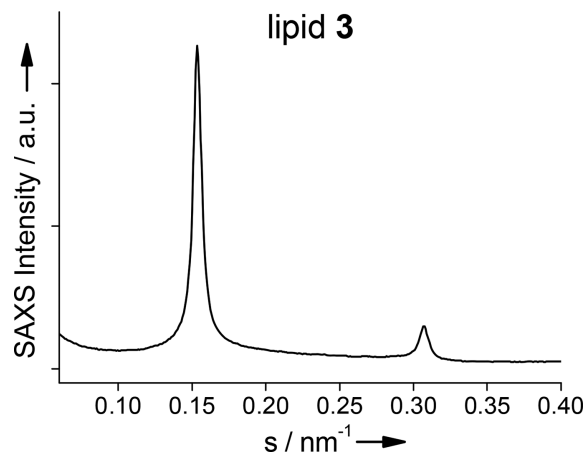

Figure 6. SAXS pattern of lipid 3 at $20{ }^{\circ} \mathrm{C}$.

Upon increasing temperature, $d$ of lipid 1 decreases 432 constantly until the broad phase transition range starts at 40433 ${ }^{\circ} \mathrm{C}$. The decrease is much more drastic in the transition range 434 between 40 and $60{ }^{\circ} \mathrm{C}$ measured by DSC (Table 3). The first 435 process can be explained by hydration of headgroups already 436 discussed in the WAXS section (see section 3.2). The higher 437 the temperature the greater is the number of water molecules 438 that penetrate into the headgroup region. The following drastic 439 decrease of $d$ at higher temperature can be assigned to the 440 main phase transition (see section 3.1). The melting of the 441 chains leads to the shrinkage of the bilayers. A similar decrease 442 of $d$ can be observed at increasing temperature for lipid 2443 (Table 3). However, after the phase transition, $d$ increases 444 suddenly. Such behavior could be explained by structural 445 rearrangement of the lipids. A more plausible explanation is at 446 this point difficult to find.

447

The structurally similar lipids $\mathbf{1 - 3}$ show that a certain 448 balance in the area requirement of headgroups and tails is 449 necessary to form certain structures. While lipids $\mathbf{1}$ and $\mathbf{2}$ form 450 aggregate-like brick structures, the introduction of one double 451 bond in each chain of lipid 3 changes the curvature of the 452 bilayer. Thus, a simple lamellar structure is established. 453

The SAXS patterns of lipids $\mathbf{4}$ and $\mathbf{5}$ are presented in Figure $454 \mathrm{f7}$ 7. Lipid 4 shows mainly diffuse scattering of uncorrelated $455 \mathrm{f} 7$ bilayers (vesicular structures). The occurring Bragg peaks on 456 top are in equidistant spacing and indicate a lamellar phase 457 (Table 3). They cannot be compared to the ones of lipids 1458 and 2. The $d$ spacing is much smaller and might result from a 459

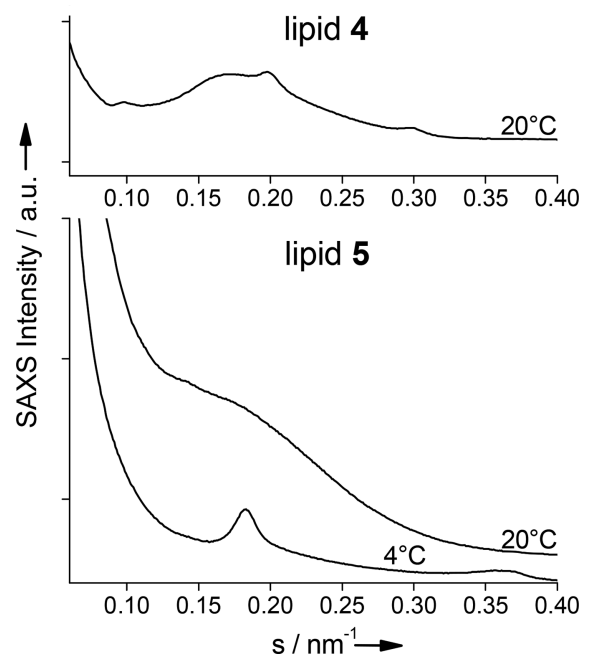

Figure 7. SAXS pattern of lipids $\mathbf{4}$ and $\mathbf{5}$ at different temperatures. 
460 single monolayer. An attempt to fit the data, as performed for 461 lipids $\mathbf{1}$ and 2, failed. Most likely this structure appears only in 462 high concentrated bulk systems where some lipid molecules 463 cannot be fully hydrated. It was shown in section 3.2 that a 464 WAXS signal of weak intensity is evidence for the presence of 465 an ordered chain lattice. It is, therefore, believed that this 466 inhomogeneity is a lamellar phase which is in the gel state. 467 However, no phase transition could be detected in the highly 468 diluted system in the DSC experiment.

469 Lipid 5 shows a lamellar phase with equidistant spacing of 470 the Bragg peaks at $4{ }^{\circ} \mathrm{C}$. Upon heating to $20{ }^{\circ} \mathrm{C}$, the system 471 exhibits pure diffuse scattering. The interbilayer positional 472 correlations are lost. This so-called unbinding transition has 473 been observed before and usually coincides with the $\mathrm{L}_{\mathrm{B}^{\prime}} \rightarrow \mathrm{L}_{\alpha}$ 474 transition (as in the present case). ${ }^{40}$ It is explained by a drastic 475 reduction of the bending rigidity upon entering the $\mathrm{L}_{\alpha}$-phase. 476 The stronger steric repulsion leads to the unbinding of the 477 stacks.

478 By the comparison of lipids 4 and 5 with the first ones, 479 similar structural relationships can be derived. Vesicular 480 structures are found for lipid $\mathbf{4}$ due to differently sized 481 hydrophilic and hydrophobic parts. The membranes will bend 482 to a high degree, and continuous bilayers cannot be formed. 483 Only with the introduction of one double bond into lipid $\mathbf{5}$ are 484 the chains able to sterically compensate for the size of the 485 headgroup and a lamellar phase is established.

486 A completely different structure is found for lipid 6 at $20{ }^{\circ} \mathrm{C}$ 487 (Figure 8). The peaks can be indexed in the order $\sqrt{2}, \sqrt{4}$,

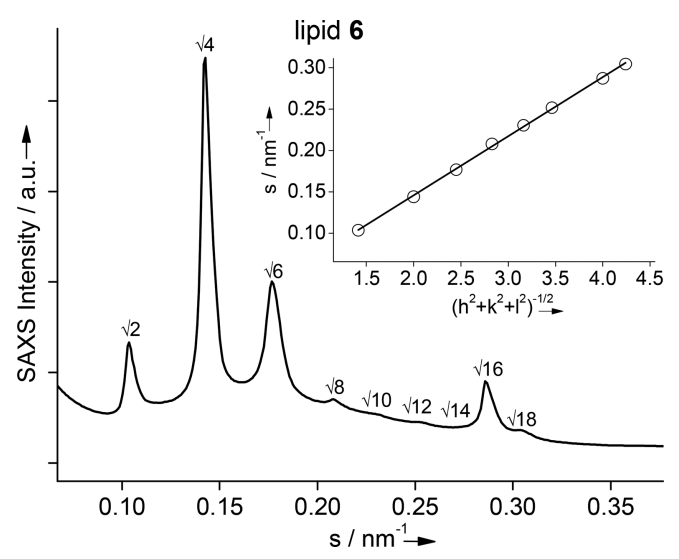

Figure 8. SAXS pattern of lipid 6 at $20^{\circ} \mathrm{C}$. The inlay shows the linear fit of the peak positions $s$ as a function of $\left(h^{2}+k^{2}+l^{2}\right)^{1 / 2}$.

$488 \sqrt{ } 6, \sqrt{ } 8, \sqrt{ } 10, \sqrt{ } 12, \sqrt{ } 16$, and $\sqrt{ } 18$. This pattern is typical 489 for a body-centered cubic (bcc) lattice (=Im $3 m$ symmetry). ${ }^{24}$ 490 The inlay of Figure 8 shows the linear fit of the Miller indices 491 as a function of $s$ with a correlation coefficient of $r=0.9992$. It 492 should be mentioned that the reflection for $\sqrt{ } 14$ was not 493 found (intensity already too low). The lattice parameter $a$ 494 obtained from the linear fit is $13.42 \mathrm{~nm}$.

495 Finally, the structural assemblies of lipids $\mathbf{1 - 6}$ shall be 496 discussed with respect to transfection efficiencies. It is eye497 catching that lipids $\mathbf{1}$ and $\mathbf{2}$ show very similar structures with 498 very large $d$ values. The unusual, brick-like structures seem to 499 be disadvantageous for mixtures with colipids. In contrast, 500 lipids 3-6 form completely different structures. All of them 501 were able to establish colloidal systems with particle sizes 502 below $200 \mathrm{~nm}$ in a mixture with helper lipids (DOPE or 503 cholesterol) and later on in complexes with DNA. ${ }^{10}$ Never- theless, only the lipoplexes of lipid 6, a lipid which forms cubic 504 mesophases, and lipid 3, a lipid which prefers lamellar 505 assembling, are highly effective. ${ }^{10}$ For a better understanding, 506 we focus now on these two lipids.

3.4. SAXS Patterns of Lipid Mixtures and Complexes 508 with DNA. In order to correlate transfection experiments with 509 supramolecular structures of lipoplexes, closely related model 510 systems are investigated. Lipid mixtures with colipids and 511 complexes with DNA are chosen exemplarily according to 512 Wölk et al. ${ }^{10}$ All systems under investigation show outstanding 513 transfection results in in vitro cell culture models even in 514 comparison with commercially available reagents. ${ }^{10}$ In Table 4, 515 t4 structures and lattice parameters are presented.

Table 4. Structure and Interlamellar Repeat Distance $d$ (if Lamellar) or Lattice Parameter $a$ of the Unit Cell (if Hexagonal or Cubic) of Lipid Mixtures with and without DNA at $20{ }^{\circ} \mathrm{C}$ Obtained from the X-ray Data ${ }^{a}$

\begin{tabular}{llc}
\multicolumn{1}{c}{ sample } & \multicolumn{1}{c}{ structure } & $d / a[\mathrm{~nm}]$ \\
lipid 3: DOPE 1:2 & $\mathrm{L}_{\alpha}$ & 5.92 \\
& $\mathrm{H}_{\mathrm{II}}$ & 7.33 \\
lipid 3: DOPE 1:2 (after heating to $80{ }^{\circ} \mathrm{C}$ ) & $\mathrm{L}_{\alpha}$ & 5.87 \\
& $\mathrm{H}_{\mathrm{II}}$ & 7.34 \\
& Im3m & 16.5 \\
lipid 3: DOPE 1:2 + DNA & $\mathrm{L}_{\alpha}$ (DNA) & 7.21 \\
& Im3m (DNA) & 20.6 \\
lipid 6: DOPE 2:1 & $\mathrm{L}_{\alpha}$ & 6.41 \\
lipid 6: DOPE 2:1 + DNA & $\mathrm{L}_{\alpha}$ & 6.45 \\
& $\mathrm{~L}_{\alpha}$ (DNA) & 7.41
\end{tabular}

${ }^{a} \mathrm{~L}_{\alpha}$ represents the lamellar, liquid-crystalline phase and $\mathrm{H}_{\mathrm{II}}$ represents the inverted hexagonal phase.

Figure 9 shows the mixture of lipid 3 with DOPE in a ratio $517 \mathrm{fg}$ of $1: 2(\mathrm{n} / \mathrm{n})$ directly after 7 days of storage at $4{ }^{\circ} \mathrm{C}$ (=thermal 518 equilibrium) and $5 \mathrm{~min}$ after heating once to $80{ }^{\circ} \mathrm{C}$ and 519 cooling to $20{ }^{\circ} \mathrm{C}$ with a cooling rate of $2 \mathrm{~K} \mathrm{~min}^{-1}$. In both 520 cases, a complex SAXS pattern is found. In thermal 521

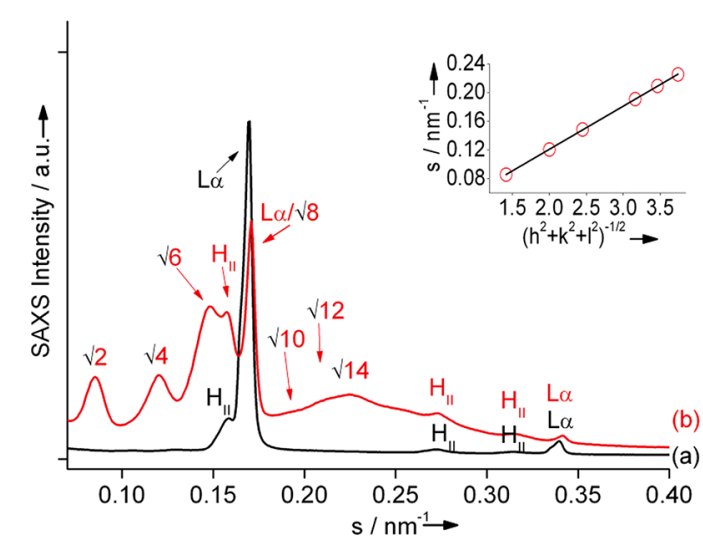

Figure 9. SAXS patterns of lipid 3 in $1: 2$ ratio $(\mathrm{n} / \mathrm{n})$ with DOPE before (a) and after (b) heating to $80{ }^{\circ} \mathrm{C}\left(2 \mathrm{Kmin}^{-1}\right)$, cooling (2 $\mathrm{Kmin}^{-1}$ ), and a waiting period of $5 \mathrm{~min}$ at $20^{\circ} \mathrm{C}$. The lamellar phase of lipid 3 and DOPE is indexed with $\mathrm{L}_{\alpha}$. The peaks of the inverted hexagonal phase of pure DOPE are marked with $\mathrm{H}_{\mathrm{II}}$. The additional cubic phase with $\operatorname{Im} 3 m$ symmetry is indexed using square roots. Curves are shifted vertically and the intensity of (b) was increased by a factor of 2 for clarity. The inlay shows the linear fit of the peak positions $s$ as a function of $\left(h^{2}+k^{2}+l^{2}\right)^{1 / 2}$ resulting from pattern (b). 
522 equilibrium, two coexisting phases are present. A lamellar $\mathrm{L}_{\alpha^{-}}$ 523 phase (halo in WAXS region) with equidistant spacings of the 524 peaks can be seen. The interlamellar repeat distance is $\sim 0.6$ $525 \mathrm{~nm}$ smaller than that found for the pure lipid 3. This can be 526 assigned to the interplay of lipid 3 with DOPE. The chains of 527 both lipids are in a liquid-like state; therefore, the thickness of 528 the hydrophobic part does not change so dramatically. 529 However, interactions between the headgroups can reduce 530 the effective extension of the hydrophilic region. A change in 531 the interlayer water thickness is also possible. The SAXS 532 patterns show additional peaks with low intensities which are 533 typical for the $\mathrm{H}_{\mathrm{II}}$-phase of pure DOPE in water. ${ }^{41}$ It can be 534 concluded that a maximum miscibility of DOPE is reached in 535 the lamellar phase of lipid 3. Above a certain DOPE ratio, the $536 \mathrm{H}_{\mathrm{II}}$-phase of phase-separated DOPE is formed additionally.

537 If the lipid mixture is heated to $80{ }^{\circ} \mathrm{C}$ and immediately 538 cooled to $20^{\circ} \mathrm{C}$, the same two phases can be found after $5 \mathrm{~min}$ 539 (Figure 9). However, the peaks representing the $\mathrm{L}_{\alpha}$-phase are 540 strongly diminished in intensity and a third phase appears. This 541 phase can be indexed as a cubic phase with $\operatorname{Im} 3 m$ symmetry 542 (see inlay of Figure 9). Only six peaks were found, but their 543 positions are in almost perfect correlation with the linear fit $(r$ $544=0.99995)$. Increasing temperature leads to a change of 545 miscibility of DOPE with lipid 3 . The fast cooling process 546 allows the conservation of this state (some phase-separated 547 DOPE is still present), and the cubic phase can be seen.

548 The $\operatorname{Im} 3 m$ phase becomes especially interesting if complexes 549 of the lipid 3:DOPE mixture $(1: 2 \mathrm{n} / \mathrm{n})$ and DNA (N/P 4:1) 550 are considered. This model system represents a real trans551 fection system that turned out to be promising in the presence 552 and absence of serum. ${ }^{10}$ Figure 10 shows the corresponding 553 SAXS pattern.

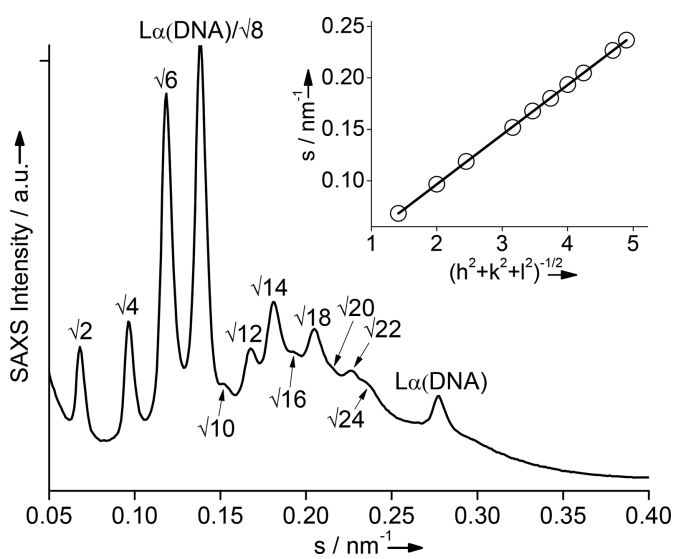

Figure 10. SAXS pattern of lipid 3 in 1:2 ratio $(n / n)$ with DOPE complexed with DNA (N/P 4:1) at $20{ }^{\circ} \mathrm{C}$. The lamellar $\mathrm{L}_{\alpha}$-phase built by lipid 3, DOPE, and DNA is indexed with $\mathrm{L}_{\alpha}$ (DNA). The coexisting cubic phase with Im $3 m$ symmetry complexed with DNA is indexed using square roots. The inlay shows the linear fit of the peak positions $s$ as a function of $\left(h^{2}+k^{2}+l^{2}\right)^{1 / 2}$ of the cubic phase.

554 Again two coexisting phases are found: (i) A lamellar $\mathrm{L}_{\alpha^{-}}$ 555 phase (halo in WAXS region) represented by a peak pattern of 556 equidistant spacing. The interlamellar repeat distance $d$ is 557 increased by $1.3 \mathrm{~nm}$ if compared to that of the system without 558 DNA (Table 4). (ii) A cubic phase with $\operatorname{Im} 3 m$ symmetry. It 559 was possible to assign 10 peaks and fit $s$ versus the Miller 560 indices linearly $(r=0.99991)$. The lattice parameter of the unit 561 cell $a$ is increased by $4.1 \mathrm{~nm}$ (Table 4). It can be concluded that the expansion is caused in both cases by the integration of 562 DNA strands. In the case of lamellar phases, as usual, $d$ does 563 not increase enough to accommodate DNA with a diameter of 564 $\sim 2 \mathrm{~nm}$ (B-form). ${ }^{12,42}$ The additionally needed space is gained 565 in a different way (e.g., the squeezing out of water between the 566 bilayers, changing the tilting of the lipid chains, or disordering 567 of the chains). The DNA-bearing cubic phase with $\operatorname{Im} 3 m 568$ symmetry is a special lipoplex structure which needs additional 569 attention. Cubic lipoplexes with different symmetries are rarely 570 described after interaction of small siRNA with cationic lipid 571 mixtures. ${ }^{43-45}$ That long DNA molecules induce the formation 572 of cubic lipoplexes is a rather new observation: Bilalov et al. 573 described a DNA-bearing cubic phase with Ia3d symmetry. ${ }^{15} 574$ McLoughin et al. described a cubic lipoplex with Pm3m 575 symmetry for cationic surfactant/DNA mixtures which exhibit 576 low effectivity and are highly toxic in mammalian cells, 577 comparable to the systems described by Zhou et al. ${ }^{17,18}$ Cubic 578 phases are intermediates in membrane fusion processes. ${ }^{43}$ In 579 literature, it is proposed that cubic phases have efficient 580 fusogenic properties which are independent of the membrane 581 charge density and consequently trigger endosomal es- 582 cape. $^{44,45,49}$ However, a generalized connection between 583 cubic lipoplex structures and high transfection efficiency does 584 not exist. Koynova et al. described that several lipoplex 585 structures undergo phase transitions toward cubic phases when 586 interacting with model biomembranes. ${ }^{46}$ Our findings suggest 587 that combined lamellar and cubic lipoplexes, which are formed 588 before the contact of the lipoplexes with cellular lipids, can also 589 trigger transfection efficiency. Leal et al. were also able to 590 connect siRNA-loaded cubic lipoplex structures with an 591 efficient nucleic acid delivery. ${ }^{50}$ Nevertheless, recently, we 592 demonstrated that a lipoplex formulation with a lamellar 593 superstructure was more efficient than a lipoplex with a cubic 594 structure. ${ }^{51}$ Obviously, more factors (for instance lipoplex 595 stability, lipoplex charge, and binding strength between lipids 596 and DNA) than the fusiogenic potential of cubic phases affect 597 the transfection efficiency.

The coexistence of both phases might be decisive 599 considering the transfection results with and without serum. 600 It is shown that $\mathrm{L}_{\alpha}{ }^{\mathrm{c}}$-phases are effective gene vectors for 601 applications with serum and in vivo studies. . $^{2,47,48}$ It is 602 proposed that the tight packing of the lamellar structures 603 prevents the dissociation of lipoplexes enforced by molecules 604 in biological fluids. In contrast, in the absence of serum, usually 605 $\mathrm{H}_{\text {II }}$ or cubic phases are more effective gene vectors. ${ }^{1,2} 606$ Therefore, coexistence of two phases could be the reason for 607 effective gene transfer with and without serum. In each case, 608 one phase will be more effective. One possible scenario for the 609 combination of both structures is a cubic structure embedded 610 in a lamellar one (see Figure 12).

611

Lipid 6 shows especially high transfection results in a 2:1 612 mixture $(\mathrm{n} / \mathrm{n})$ with DOPE complexed with DNA (N/P 613 1.67:1). ${ }^{10}$ The SAXS patterns of the model system with and 614 without DNA are shown in Figure 11.

In the case of the lipid mixture, an equidistant peak pattern 616 indicates that a lamellar $\mathrm{L}_{\alpha}$-phase is formed (halo in WAXS 617 region). The addition of DOPE to lipid 6 transforms the lattice 618 structure from cubic to lamellar. The same SAXS peak pattern 619 is present in the complex with DNA. Furthermore, a second set 620 of peaks with high scattering intensities appears and indicates 621 the integration of DNA between bilayers. The interlamellar 622 repeat distance is increased by $1.0 \mathrm{~nm}$ compared to that of the 623 pure lipid mixture. It can be concluded that a small amount of 624 


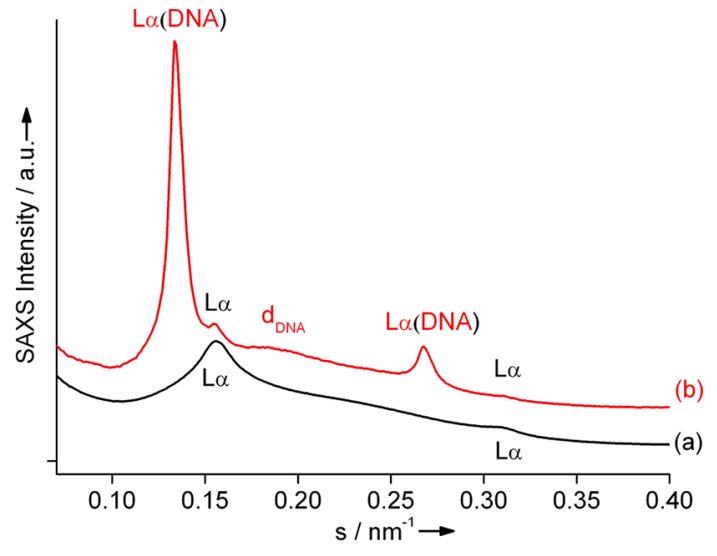

Figure 11. SAXS patterns of lipid 6 in $2: 1$ ratio $(n / n)$ with DOPE at $20{ }^{\circ} \mathrm{C}$ (a) and in complex with DNA (N/P 1.67:1) (b) at $20{ }^{\circ} \mathrm{C}$. Lamellar phases of the lipids without DNA are indexed with $\mathrm{L}_{\alpha}$. The lamellar phase containing DNA is indexed with $\mathrm{L}_{\alpha}$ (DNA). The broad scattering peak marked with $d_{\mathrm{DNA}}$ represents the one-dimensional repeat distance of aligned DNA strands.

625 empty $\mathrm{L}_{\alpha}$-phase is in coexistence with a $\mathrm{L}_{\alpha}$-phase with DNA 626 strands intercalated between the layers. An additional broad 627 peak with small intensity appears in the pattern for the 628 complex with DNA. This peak represents the one-dimensional 629 interaxial repeat distance of aligned DNA strands and is most 630 commonly found in comparable systems. In this case, $d_{\text {DNA }}$ was 631 calculated to be $5.18 \mathrm{~nm}$.

\section{CONCLUSIONS}

632 The physical-chemical properties of six new lipids designed 633 for gene transfection were investigated in bulk using DSC and 634 SAXS/WAXS. The lipids can be categorized into two groups 635 with different headgroup structure: (i) lipids 1-3 and (ii) 636 lipids 4-6. In these groups, the saturation degree of the chains 637 is varied.

638 In (i), lipids 1 (saturated) and 2 (one double bond) are 639 strongly self-associating. Interactions between lipid chains and 640 between headgroups are found. Lipid $\mathbf{1}$ is in the subgel state, 641 and lipid $\mathbf{2}$ is in the gel state at room temperature with strongly 642 reduced mobility compared to liquid-crystalline phases. Both 643 lipids arrange in brick-like structures. This disadvantageous 644 behavior explains the low affinity to helper lipids which were 645 not incorporated. Therefore, both lipids are not suited for gene 646 transfer.

647 Due to insertion of one double bond in each chain, the 648 phase transition temperature (from gel to liquid-crystalline 649 state) of lipid 3 is decreased below room temperature and a 650 lamellar phase is formed. Increased fluidity in the liquid651 crystalline state leads to a possible incorporation of 652 zwitterionic helper lipids. In mixtures with DOPE, the lamellar 653 structure is preserved and only upon heating are changes to 654 coexisting lamellar and cubic structures observed. The cubic 655 one is, therefore, a metastable mesophase. The addition of 656 DNA leads to similar coexisting phases with larger lattice 657 parameters due to the incorporated DNA. The often described $658 \mathrm{~L}_{\alpha}{ }^{c}$ lipoplex structure and a rarely observed $\operatorname{Im} 3 m \mathrm{Q}_{\alpha}{ }^{\mathrm{c}}$ lipoplex 659 structure occur. Because the coexistence is found without 660 heating, DNA has a stabilizing effect on the assembly in cubic 661 structures. The alteration of the self-assembled supramolecular 662 structure of lipid 3 with the addition of DOPE and after 663 interaction with DNA is summarized in Figure 12. The high gene transfer activity of lipid 3 with DOPE, in the presence and 664 absence of serum, might be based on the coexistence of these 665 two phases.

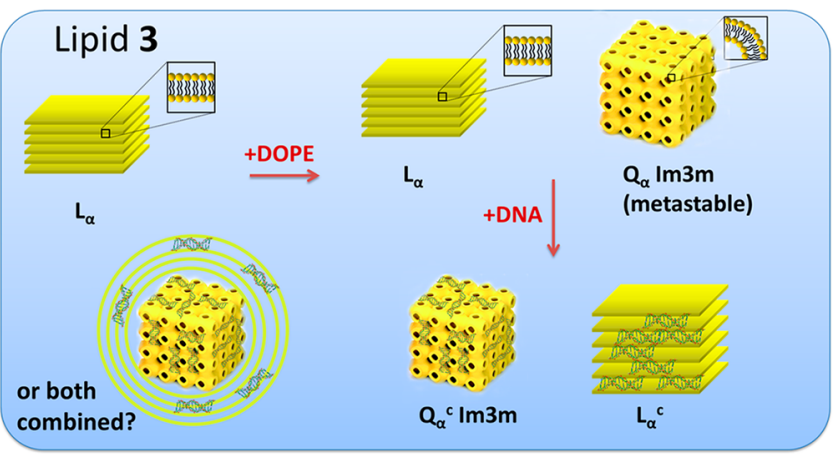

\section{Lipid 6}

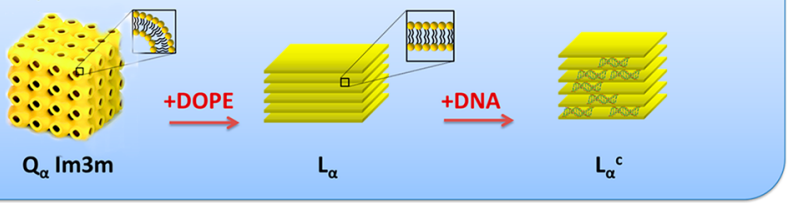

Figure 12. Schematic illustration of the alteration of the supramolecular self-assembling structures of lipid 3 and lipid 6 after addition of DOPE and interaction with DNA.

In (ii), interactions of chains are disrupted by larger 667 headgroups which need more space. Therefore, all lipids are 668 in the liquid-crystalline state at room temperature. The fluidity 669 and mobility of the lipids is increased, and the incorporation of 670 noncharged or zwitterionic helper lipids is possible. Interest- 671 ingly, lipid 6 with two double bonds shows again the highest 672 transfection rates. The pure substance forms a cubic phase with 673 $\operatorname{Im} 3 m$ symmetry, while in a mixture with DOPE and DNA, 674 lamellar phases are found. Therefore, the results of Koynova at 675 al. ${ }^{1}$ showing that lamellar phases can be efficient gene carriers, 676 like $\mathrm{H}_{\text {II }}$ phases, are supported. In contrast to lipid $3\left(\mathrm{~L}_{\alpha} \rightarrow \mathrm{L}_{\alpha} / 677\right.$ $\mathrm{Q}_{\alpha}$ (metastable) $\rightarrow \mathrm{L}_{\alpha}{ }^{\mathrm{c}} / \mathrm{Q}_{\alpha}{ }^{\mathrm{c}} \operatorname{Im} 3 \mathrm{~m}$ ), the self-assembling 678 behavior of lipid 6 changes after adding DOPE and complexing 679 DNA in the following way: $\mathrm{Q}_{\alpha} \operatorname{Im} 3 m \rightarrow \mathrm{L}_{\alpha} \rightarrow \mathrm{L}_{\alpha}{ }^{\mathrm{c}}$ (see Figure 680 12).

It can be concluded that double bonds are crucial for the 682 phase structures of the presented lipids and for transfection 683 efficiency. Saturated lipids especially show disadvantageous 684 rigid molecular assemblies. With increasing degree of 685 unsaturation, significant structural changes occur together 686 with increasing gene transfer activity. Furthermore, DNA can 687 change the supramolecular self-assembly in an unpredictable 688 manner.

\section{AUTHOR INFORMATION}

\section{Corresponding Authors}

*E-mail: brezesinski@mpikg.mpg.de.

*E-mail: christian.woelk@pharmazie.uni-halle.de.

Christian Wölk: 0000-0002-8067-7307

The authors declare no competing financial interest. 
698

\section{ACKNOWLEDGMENTS}

699 We thank the personnel of the A2 beamline at HASYLAB at 700 DESY in Hamburg, Germany, for professional support at all 701 times. We thank G. Pabst for providing for us the GAP 702 software. Katharina Koch is thanked for assistance with sample 703 preparation. This work was supported by the Max Planck 704 Society.

\section{$705 \square$ REFERENCES}

706 (1) Koynova, R.; Tenchov, B. Cationic phospholipids: structure707 transfection activity relationships. Soft Matter 2009, 5 (17), 31877083200 .

709 (2) Ma, B.; Zhang, S.; Jiang, H.; Zhao, B.; Lv, H. Lipoplex 710 morphologies and their influences on transfection efficiency in gene 711 delivery. J. Controlled Release 2007, 123 (3), 184-194.

712 (3) Ewert, K. K.; Zidovska, A.; Ahmad, A.; Bouxsein, N. F.; Evans, 713 H. M.; McAllister, C. S.; Samuel, C. E.; Safinya, C. R. Cationic 714 Liposome-Nucleic Acid Complexes for Gene Delivery and Silencing: 715 Pathways and Mechanisms for Plasmid DNA and siRNA. In Nucleic 716 Acid Transfection; Bielke, W., Erbacher, C., Eds.; Springer Berlin 717 Heidelberg: Berlin, 2010; pp 191-226.

718 (4) Zuhorn, I. S.; Oberle, V.; Visser, W. H.; Engberts, J. B. F. N.; 719 Bakowsky, U.; Polushkin, E.; Hoekstra, D. Phase Behavior of Cationic 720 Amphiphiles and Their Mixtures with Helper Lipid Influences 721 Lipoplex Shape, DNA Translocation, and Transfection Efficiency. 722 Biophys. J. 2002, 83 (4), 2096-2108.

723 (5) Zhi, D.; Zhang, S.; Wang, B.; Zhao, Y.; Yang, B.; Yu, S. 724 Transfection Efficiency of Cationic Lipids with Different Hydro725 phobic Domains in Gene Delivery. Bioconjugate Chem. 2010, 21 (4), $726563-577$.

727 (6) Bajaj, A.; Kondaiah, P.; Bhattacharya, S. Gene Transfection 728 Efficacies of Novel Cationic Gemini Lipids Possessing Aromatic 729 Backbone and Oxyethylene Spacers. Biomacromolecules 2008, 9 (3), 730 991-999.

731 (7) Felgner, J. H.; Kumar, R.; Sridhar, C. N.; Wheeler, C. J.; Tsai, Y. 732 J.; Border, R.; Ramsey, P.; Martin, M.; Felgner, P. L. Enhanced gene 733 delivery and mechanism studies with a novel series of cationic lipid 734 formulations. J. Biol. Chem. 1994, 269 (4), 2550-2561.

735 (8) Pelisek, J.; Gaedtke, L.; DeRouchey, J.; Walker, G. F.; Nikol, S.; 736 Wagner, E. Optimized lipopolyplex formulations for gene transfer to 737 human colon carcinoma cells under in vitro conditions. J. Gene Med. 738 2006, 8 (2), 186-197.

739 (9) Heinze, M.; Brezesinski, G.; Dobner, B.; Langner, A. Novel 740 Cationic Lipids Based on Malonic Acid Amides Backbone: Trans741 fection Efficacy and Cell Toxicity Properties. Bioconjugate Chem. 742 2010, 21 (4), 696-708.

743 (10) Wölk, C.; Heinze, M.; Kreideweiß, P.; Dittrich, M.; Brezesinski, 744 G.; Langner, A.; Dobner, B. Synthesis and DNA transfection 745 properties of new head group modified malonic acid diamides. Int. 746 J. Pharm. (Amsterdam, Neth.) 2011, 409 (1), 46-56.

747 (11) Wölk, C.; Janich, C.; Bakowsky, U.; Langner, A.; Brezesinski, G. 748 Malonic acid based cationic lipids - The way to highly efficient DNA749 carriers. Adv. Colloid Interface Sci. 2017, 248, 20-34.

750 (12) Rädler, J. O.; Koltover, I.; Salditt, T.; Safinya, C. R. Structure of 751 DNA-Cationic Liposome Complexes: DNA Intercalation in Multi752 lamellar Membranes in Distinct Interhelical Packing Regimes. Science 753 1997, 275 (5301), 810-814.

754 (13) Koltover, I.; Salditt, T.; Rädler, J. O.; Safinya, C. R. An Inverted 755 Hexagonal Phase of Cationic Liposome-DNA Complexes Related to 756 DNA Release and Delivery. Science 1998, 281 (5373), 78-81.

757 (14) Ewert, K. K.; Evans, H. M.; Zidovska, A.; Bouxsein, N. F.; 758 Ahmad, A.; Safinya, C. R. A Columnar Phase of Dendritic Lipid759 Based Cationic Liposome-DNA Complexes for Gene Delivery: 760 Hexagonally Ordered Cylindrical Micelles Embedded in a DNA 761 Honeycomb Lattice. J. Am. Chem. Soc. 2006, 128 (12), 3998-4006. 762 (15) Bilalov, A.; Olsson, U.; Lindman, B. A cubic DNA-lipid 763 complex. Soft Matter 2009, 5 (20), 3827-3830.
(16) Koynova, R. Lipid Phases Eye View to Lipofection. Cationic 764 Phosphatidylcholine Derivatives as Efficient DNA Carriers for Gene 765 Delivery. Lipid Insights 2008, 2, LPI.S864.

(17) McLoughlin, D.; Impéror-Clerc, M.; Langevin, D. A New 767 Cubic Phase Containing DNA and a Surfactant. ChemPhysChem 768 2004, 5 (10), 1619-1623.

(18) Zhou, S.; Liang, D.; Burger, C.; Yeh, F.; Chu, B. Nanostructures 770 of Complexes Formed by Calf Thymus DNA Interacting with 771 Cationic Surfactants. Biomacromolecules 2004, 5 (4), 1256-1261. 772 (19) Šmisterová, J.; Wagenaar, A.; Stuart, M. C. A.; Polushkin, E.; 773 ten Brinke, G.; Hulst, R.; Engberts, J. B. F. N.; Hoekstra, D. Molecular 774 Shape of the Cationic Lipid Controls the Structure of Cationic Lipid/ 775 Dioleylphosphatidylethanolamine-DNA Complexes and the Efficiency 776 of Gene Delivery. J. Biol. Chem. 2001, 276 (50), 47615-47622. 777 (20) Koch, M. H. J.; Bordas, J. X-ray diffraction and scattering on 778 disordered systems using synchrotron radiation. Nucl. Instrum. 779 Methods Phys. Res. 1983, 208 (1), 461-469.

(21) Boulin, C.; Kempf, R.; Koch, M. H. J.; McLaughlin, S. M. Data 781 appraisal, evaluation and display for synchrotron radiation experi- 782 ments: Hardware and software. Nucl. Instrum. Methods Phys. Res., Sect. 783 A 1986, 249 (2), 399-407.

(22) Boulin, C. J.; Kempf, R.; Gabriel, A.; Koch, M. H. J. Data 785 acquisition systems for linear and area X-ray detectors using delay line 786 readout. Nucl. Instrum. Methods Phys. Res., Sect. A 1988, 269 (1), 312- 787 320.

(23) Rapp, G.; Gabriel, A.; Dosière, M.; Koch, M. H. J. A dual 789 detector single readout system for simultaneous small (SAXS) and 790 wide-angle X-ray (WAXS) scattering. Nucl. Instrum. Methods Phys. 791 Res., Sect. A 1995, 357 (1), 178-182.

(24) Lindblom, G.; Rilfors, L. Cubic phases and isotropic structures 793 formed by membrane lipids - possible biological relevance. Biochim. 794 Biophys. Acta, Rev. Biomembr. 1989, 988 (2), 221-256. 795

(25) Dörfler, H.-D.; Brezesinski, G.; Miethe, P. Phase diagrams of 796 pseudo-binary phospholipid systems I. Influence of the chain length 797 differences on the miscibility properties of cephaline/cephaline/water 798 systems. Chem. Phys. Lipids 1988, 48 (3), 245-254.

799

(26) Nuhn, P.; Brezesinski, G.; Dobner, B.; Förster, G.; Gutheil, M.; 800 Dörfler, H.-D. Synthesis, calorimetry, and X-ray diffraction of lecithins 801 containing branched fatty acid chains. Chem. Phys. Lipids 1986, 39802 (3), 221-236.

803

(27) Dittrich, M.; Heinze, M.; Wölk, C.; Funari, S. S.; Dobner, B.; 804 Möhwald, H.; Brezesinski, G. Structure-Function Relationships of 805 New Lipids Designed for DNA Transfection. ChemPhysChem 2011, 806 12 (12), 2328-2337.

(28) Cevc, G. How membrane chain-melting phase-transition 808 temperature is affected by the lipid chain asymmetry and degree of 809 unsaturation: an effective chain-length model. Biochemistry 1991, 30810 (29), 7186-7193.

(29) Bennett, C. F.; Mirejovsky, D.; Crooke, R. M.; Tsai, Y. J.; 812 Felgner, J.; Sridhar, C. N.; Wheeler, C. J.; Felgner, P. L. Structural 813 Requirements for Cationic Lipid Mediated Phosphorothioate 814 Oligonucleotides Delivery to Cells in Culture. J. Drug Targeting 815 1998, 5 (3), 149-162.

816

(30) Nguyen, J. T.; Inouye, H.; Baldwin, M. A.; Fletterick, R. J.; 817 Cohen, F. E.; Prusiner, S. B.; Kirschner, D. A. X-ray Diffraction of 818 Scrapie Prion Rods and PrP Peptides. J. Mol. Biol. 1995, 252 (4), 819 $412-422$.

820

(31) Hartgerink, J. D.; Granja, J. R.; Milligan, R. A.; Ghadiri, M. R. 821 Self-Assembling Peptide Nanotubes. J. Am. Chem. Soc. 1996, 118 (1), 822 $43-50$.

823

(32) Serpell, L. C. Alzheimer's amyloid fibrils: structure and 824 assembly. Biochim. Biophys. Acta, Mol. Basis Dis. 2000, 1502 (1), 825 $16-30$.

(33) Dittrich, M.; Böttcher, M.; Oliveira, J. S. L.; Dobner, B.; 827 Möhwald, H.; Brezesinski, G. Physical-chemical characterization of 828 novel cationic transfection lipids and the binding of model DNA at 829 the air-water interface. Soft Matter 2011, 7 (21), 10162-10173. 830 
831 (34) Kuzmenko, I.; Kaganer, V. M.; Leiserowitz, L. Packing of 832 Hydrocarbon Chains and Symmetry of Condensed Phases in 833 Langmuir Monolayers. Langmuir 1998, 14 (14), 3882-3888.

834 (35) Pabst, G. Global Properties of biomimetic membranes: 835 Perspectives on molecular features. Biophys. Rev. Lett. 2006, 01, $83657-84$.

837 (36) Funari, S. S.; Rapp, G.; Richter, F. Double-bilayer: a new phase 838 formed by lysophospholipids and the corresponding fatty acid. Quim. 839 Nova 2009, 32, 908-912.

840 (37) Bouwstra, J. A.; Gooris, G. S.; van der Spek, J. A.; Bras, W. 841 Structural Investigations of Human Stratum Corneum by Small-Angle 842 X-Ray Scattering. J. Invest. Dermatol. 1991, 97 (6), 1005-1012.

843 (38) Pabst, G.; Rappolt, M.; Amenitsch, H.; Laggner, P. Structural 844 information from multilamellar liposomes at full hydration: Full q845 range fitting with high quality x-ray data. Phys. Rev. E: Stat. Phys., 846 Plasmas, Fluids, Relat. Interdiscip. Top. 2000, 62 (3), 4000-4009.

847 (39) Pabst, G.; Koschuch, R.; Pozo-Navas, B.; Rappolt, M.; Lohner, $848 \mathrm{~K}$.; Laggner, P. Structural analysis of weakly ordered membrane 849 stacks. J. Appl. Crystallogr. 2003, 36 (6), 1378-1388.

850 (40) Pozo-Navas, B.; Raghunathan, V. A.; Katsaras, J.; Rappolt, M.; 851 Lohner, K.; Pabst, G. Discontinuous Unbinding of Lipid Multi852 bilayers. Phys. Rev. Lett. 2003, 91 (2), 028101.

853 (41) Turner, D. C.; Gruner, S. M. X-ray diffraction reconstruction of 854 the inverted hexagonal (HII) phase in lipid-water systems. 855 Biochemistry 1992, 31 (5), 1340-1355.

856 (42) Podgornik, R.; Rau, D. C.; Parsegian, V. A. The action of 857 interhelical forces on the organization of DNA double helixes: 858 fluctuation-enhanced decay of electrostatic double-layer and hydra859 tion forces. Macromolecules 1989, 22 (4), 1780-1786.

860 (43) Dan, N.; Danino, D. Structure and kinetics of lipid-nucleic 861 acid complexes. Adv. Colloid Interface Sci. 2014, 205, 230-239.

862 (44) Martínez-Negro, M.; Kumar, K.; Barrán-Berdón, A. L.; Datta, 863 S.; Kondaiah, P.; Junquera, E.; Bhattacharya, S.; Aicart, E. Efficient 864 Cellular Knockdown Mediated by siRNA Nanovectors of Gemini 865 Cationic Lipids Having Delocalizable Headgroups and Oligo866 Oxyethylene Spacers. ACS Appl. Mater. Interfaces 2016, 8 (34), 867 22113-22126.

868 (45) Kim, H.; Leal, C. Cuboplexes: Topologically Active siRNA 869 Delivery. ACS Nano 2015, 9 (10), 10214-10226.

870 (46) Koynova, R.; Tenchov, B.; MacDonald, R. C. Nonlamellar 871 Phases in Cationic Phospholipids, Relevance to Drug and Gene 872 Delivery. ACS Biomater. Sci. Eng. 2015, 1 (3), 130-138.

873 (47) Gao, X.; Kim, K.-S.; Liu, D. Nonviral gene delivery: What we 874 know and what is next. AAPS J. 2007, 9 (1), E92-E104.

875 (48) Tros de Ilarduya, C.; Sun, Y.; Düzgüneş, N. Gene delivery by 876 lipoplexes and polyplexes. Eur. J. Pharm. Sci. 2010, 40 (3), 159-170. 877 (49) Motta, S.; Rondelli, V.; Cantu, L.; Del Favero, E.; Aureli, M.; 878 Pozzi, D.; Caracciolo, G.; Brocca, P. What the cell surface does not 879 see: The gene vector under the protein corona. Colloids Surf., B 2016, $880141,170-178$.

881 (50) Leal, C.; Bouxsein, N. F.; Ewert, K. K.; Safinya, C. R. Highly 882 efficient gene silencing activity of siRNA embedded in a nano883 structured gyroid cubic lipid matrix. J. Am. Chem. Soc. 2010, 132, 884 16841-16847.

885 (51) Tassler, S.; Pawlowska, D.; Janich, C.; Giselbrecht, J.; Drescher, 886 S.; Langner, A.; Wölk, C.; Brezesinski, G. Lysine-based amino887 functionalized lipids for gene transfection: 3D phase behaviour and 888 transfection performance. Phys. Chem. Chem. Phys. 2018, 20, 1739388917405 .

890 (52) Dörfler, H. D.; Brezesinski, G.; Miethe, P. Phase diagrams of 891 pseudo-binary phospholipid systems I. Influence of the chain length 892 differences on the miscibility properties of cephaline/cephaline/water 893 systems. Chem. Phys. Lipids 1988, 48, 245-254.

894 (53) Garidel, P.; Blume, A. Miscibility of phospholipids with 895 identical headgroups and acyl chain lengths differing by two 896 methylene units: Effects of headgroup structure and headgroup 897 charge. Biochim. Biophys. Acta, Biomembr. 1998, 1371, 83-95.

898 (54) Sackmann, H.; Demus, D. The problems of polymorphism in 899 liquid crystals. Mol. Cryst. Liq. Cryst. 1973, 21, 239-273.
(55) Platz, G.; Thunig, C.; Hoffmann, H. Iridescent phases in 900 aminoxide surfactant solutions. Interfaces in Condensed Systems, Prog. 901 Colloid Polym. Sci. 1990, 83, 167-175.

(56) Hoffmann, H. Fascinating phenomena in Surfactant Chemistry. 903 Adv. Mater. 1994, 6, 116-129. 